\title{
Efficacy and safety of glucocorticoids in the treatment of progressive muscular dystrophy in children: a systematic review and meta-analysis
}

\author{
Liang Ru, Yanan Wang, Mei Yan \\ Department of Pediatrics, The First Affiliated Hospital of Xinjiang Medical University, Urumqi, Xinjiang, China \\ Contributions: (I) Conception and design: L Ru, M Yan; (II) Administrative support: Y Wang; (III) Provision of study materials or patients: L Ru, M \\ Yan; (IV) Collection and assembly of data: All authors; (V) Data analysis and interpretation: L Ru, Y Wang; (VI) Manuscript writing: All authors; (VII) \\ Final approval of manuscript: All authors. \\ Correspondence to: Mei Yan. Department of Pediatrics, The First Affiliated Hospital of Xinjiang Medical University, No. 137, Yushan South Road, \\ Urumqi, Xinjiang, China. Email: ymxjyfy@163.com.
}

Background: Hormonal drug therapy has been widely used in clinical practice for the treatment of progressive muscular dystrophy (PMD). Glucocorticoids, as a common drug in the clinical treatment of PMD, have been reported in several clinical studies.

Methods: Chinese and English databases were respectively searched using "randomized controlled trials", "Duchenne-type myotonic dystrophy", "glucocorticoids", Prednisone", "Prednisolone", and "Methylprednisolone", and "Defibrotide" were used as search terms. The meta-analysis was performed using the RevMan 5.3 and Stata 13 software provided by the Cochrane system.

Results: this study included five randomized controlled trials, all of which described the correct randomization method. There were four detailed descriptions of hidden distribution schemes. There were four literatures using blind method. Heterogeneity analysis showed that there was some heterogeneity between the results of the mean prognostic muscle strength, walking time of 9 meters, and 4 flights of stairs climbing between the glucocorticoid-treated group (the experimental group) and the placebo group (the control group). There were no significant differences between the experimental group and the control group in average muscle strength level, walking time of 9 meters and climbing time of 4 flights of stairs ( $M D=1.77$; 95\% CI: -0.95 to $4.48 ; \mathrm{P}=0.20>0.05)$, (MD =-12.27; 95\% CI: -35.94 to $11.40 ; \mathrm{P}=0.31>0.01),(\mathrm{MD}=-3.09$; 95\% CI: -11.16 to $4.99 ; \mathrm{P}=0.45>0.05)$. In addition, glucocorticoid treatment significantly increased creatine kinase level in patients with PMD ( $\mathrm{MD}=-0.28,95 \% \mathrm{CI}:-0.57$ to $0.00 ; \mathrm{P}=0.05)$. In terms of the incidence of adverse reactions, glucocorticoid treatment significantly increased the prognostic probability of acne, rapid hair growth, and emotional irritability in PMD patients ( $\mathrm{OR}=2.40 ; 95 \% \mathrm{CI}: 1.09$ to $5.27 ; \mathrm{P}=0.03<0.05$ ), ( $\mathrm{OR}=3.05 ; 95 \% \mathrm{CI}: 1.55$ to $5.99 ; \mathrm{P}=0.001<0.05)$, ( $\mathrm{OR}=4.04 ; 95 \% \mathrm{CI}: 1.82$ to $10.63 ; \mathrm{P}=0.001<0.05)$. There was no significant difference in the incidence of prognostic depression between the experimental group and the control group (OR $=5.11 ; 95 \% \mathrm{CI}: 0.80$ to $32.79 ; \mathrm{P}=0.09>0.05)$.

Discussion: The results suggest that glucocorticoids have a significant effect on PMD patients, but to a certain extent they increase the incidence of adverse reactions in patients after treatment. However, due to the lack of complete clinical data in some ongoing studies, our conclusions may not be fully representative.

Keywords: Glucocorticoid progressive muscular dystrophy; hormone therapy; safety; meta-analysis

Submitted Sep 09, 2021. Accepted for publication Nov 12, 2021.

doi: $10.21037 / \mathrm{tp}-21-461$

View this article at: https://dx.doi.org/10.21037/tp-21-461 


\section{Introduction}

Progressive muscular dystrophy (PMD), also known as Duchenne muscular dystrophy (DMD), is a group of muscular degeneration caused by genetic mutations. The main clinical manifestations are progressive muscle atrophy with central nervous system, cardiac, skeletal, respiratory, and gastrointestinal involvement (1,2). Progressive muscular dystrophy is a chronic disease, most children around the age of 3 to 5 years of onset, gradually appear some clinical symptoms. PMD patients will appear progressive aggravation of muscle weakness, and atrophy symptoms. Children may appear convenient gait abnormalities, mainly walking sway, up and down the floor, squatting difficulties, easy to fall and so on. In addition, children with PMD may also have symptoms of eyelid relaxation and numbness of muscles outside the eye, and severe patients will gradually experience muscle atrophy or even paralysis. The onset time, progression rate, range of involvement, and severity of different types of PMD disease vary greatly. The inheritance modes include $\mathrm{X}$-linked recessive inheritance, autosomal dominant inheritance, and autosomal recessive inheritance (3). Current data suggest that the incidence of PMD is not significantly different between countries, regions, and races, with 1 case per 3,600 to 6,000 male births (4). The incidence rate in China is about $1 / 3,853$, and it is estimated that there are about 70,000 patients nationwide (5).

At present, there are very few Western medical methods for the treatment of PMD. The most prominently used in clinical practice is hormonal shock therapy, of which the more common drugs include prednisone, prednisolone, methylprednisolone diflucan, etc. Among the PMD therapeutic drugs, glucocorticoids account for a relatively large and significant effect (6).

Some studies have reported that long-term use of glucocorticoids can prolong the independent walking time of PMD patients for 2-5 years or longer, and patients can walk independently up to the age of 16 years, prolonging patients' their lives as well as improving cardiopulmonary function (7). The mechanism by which glucocorticoids treat progressive muscular dystrophy is not fully understood. It has been suggested that glucocorticoids can induce ultrastructural changes of muscle fibers in muscular dystrophy, thus affecting the related processes of endogenous inflammatory responses. For example, it can reduce the number of dendritic cells and fibroblasts and increase the number of satellite cells, thereby increasing muscle strength to a certain extent and delay the progression of PMD disease.

In contrast, glucocorticosteroids are over-indicated medications, and patients need to be evaluated for treatment benefits and potential risks, which are fully communicated with parents, and signed informed consent must be obtained prior to treatment. In addition, studies have confirmed the need for calcium, potassium, and vitamin D supplementation along with glucocorticosteroids, and that abrupt discontinuation is not advisable as it may lead to adrenocortical insufficiency (8). Daily stress doses of hydrocortisone are required when the patient has severe trauma or other severe disease of the organo-verbal system, and cortisol concentrations should be checked periodically with a corticotropin releasing hormone or pro-adrenocorticotropic hormone stimulation test until the hypothalamic-pituitary-adrenal axis returns to normal before the patient stops ceases the drug (9).

With the widespread use of glucocorticoids in the treatment of patients with clinical PMD, a comprehensive assessment of the overall efficacy and safety of all studies addressing glucocorticoid therapy for PMD in recent years is still relatively scarce. Therefore, relevant literatures on glucocorticoid treatment for PMD patients were selected for meta-analysis, to evaluate the therapeutic effect and safety of glucocorticoid treatment for PMD patients. The novel feature of this study was that it compared the effects of glucocorticoid and placebo treatment on creatine kinase in patients with common PMD disease based on the inclusion of efficacy indicators. The incidence of adverse reactions in different included literatures was classified and compared. This study provides theoretical and data support for the clinical application of glucocorticoids in PMD. We present the following article in accordance with the PRISMA reporting checklist (available at https://dx.doi. org/10.21037/tp-21-461).

\section{Methods}

\section{Literature search}

The China National Knowledge Internet (CNKI) (1979-2021.4), China Biomedical Literature Database (1994-2021.4), Cochrane Library (2005-2021.4), Medline (1948-2021.4), and Embase (1966.1-2021.4) databases were searched by computer. Relevant published randomized controlled trials on the use of microecological agents after liver surgery were searched and relevant literature in journals was hand-searched. The relevant literature involved was selected using a compound logical search Boolean logical search. 
"Randomized controlled trials", "Duchenne-type myotonic dystrophy", "glucocorticoids", "prednisone", "prednisolone", "methylprednisolone", and "diflucan" were used as search terms in the Chinese and English databases. The meta-analysis was performed according to RevMan 5.3 (https://training. cochrane.org/online-learning/core-software-cochranereviews/revman) and Stata 13 software (https://www.stata.com/ stata13/) provided by the Cochrane system.

The above search terms were freely combined, and the first screening was performed by reading the titles and abstracts of the initially searched documents to exclude those that did not meet the criteria and to identify the included documents. A second screening was performed based on the inclusion and exclusion criteria, and the included literature was traced using a search engine. Finally, a third screening was performed by reading the full texts of the included literature, and the quality of the articles was evaluated. The search date for all literature was May 14, 2021.

\section{Literature inclusion and exclusion criteria}

Inclusion criteria: (I) published randomized controlled trials of blank controls versus glucocorticoids for PMD; (II) study subjects met the diagnostic criteria for PMD; and (III) follow-up of micro-patients was greater than 3 months.

Exclusion criteria: (I) literature such as conference presentations, review articles, research reports, and lectures; (II) studies without placebo or drug control groups, nonclinical randomized controlled trials; (III) studies involving a patient follow-up time of less than 3 months; and (IV) literature with inaccessible full text, incomplete data, or duplicate publications.

\section{Observational indicators}

The study's primary efficacy measures included patients' average muscle strength, $9 \mathrm{~m}$ walk time, 4 flights of stairs climb time, and creatine kinase levels. Secondary efficacy measures included quality of life, lung function and muscle function. The measures used to assess safety include, primarily, the incidence of adverse events, such as acne, rapid hair growth, mood irritability, irritability, depression, obesity, abnormal behavior, hirsutism, appetite changes, fractures, hypertension, and cataracts.

\section{Data extraction}

Independent extraction of data was performed by two experts using Microsoft Excel (Developed by: Microsoft Corporation, Redmond, WA, USA). Consistent conclusions were obtained through discussion in cases of disagreement between the two experts. The following data was extracted for inclusion in this study: authors of the literature, study title, time of publication of the study, contact information of the author, sample size, basic characteristics of the study subjects, treatment interventions, treatment protocols, evaluation indicators, efficacy, and adverse effects. The quality of the literature was evaluated using the Cochrane Systematic Assessor's Manual 5.1.0. (https://china.cochrane. org/cochrane-china-center/research-and-evidencetranslation/systematic-reviews-and-trials).

\section{Risk of bias and quality assessment}

Two experts independently screened the literature according to the inclusion and exclusion criteria. The Cochrane Handbook Risk of Bias Assessment Tool for Randomized Controlled Trials was used to evaluate the risk of bias of the included randomized controlled trials (RCTs). Specifically, this involved whether the random assignment method was correct, whether the allocation protocol was included for concealment, whether the method was correct, whether blinding was used for study subjects, treatment protocols, study results, selective reporting of study results; and completeness of study data. The included studies were judged as "high risk of bias", "low risk of bias", and "unclear", respectively.

\section{Statistical analysis}

Statistical analysis was performed using Stata SE 12.0 (College Station, USA). The risk bias of the included references was assessed using the Bias Risk Assessment map in RevMan 5.3. $\chi^{2}$ test was used to test the heterogeneity of each included study, in which the results of continuous variables were expressed as mean difference (MD) or standardized mean difference (SMD), and the results of discontinuous variables were expressed as odds ratio (OR). All effects were expressed with $95 \%$ confidence intervals (CI). $\mathrm{P}>0.01$ and $\mathrm{I}^{2}<50 \%$ of the heterogeneity results indicated high homogeneity of the results, and the fixedeffect model was used for meta-analysis. When $\mathrm{P}<0.01$ and $\mathrm{I}^{2}>50 \%$, the heterogeneity of the results was high, and the random-effects model was used for meta-analysis. If the heterogeneity was too large, further sensitivity index should be carried out. 
Identification of studies via databases and registers
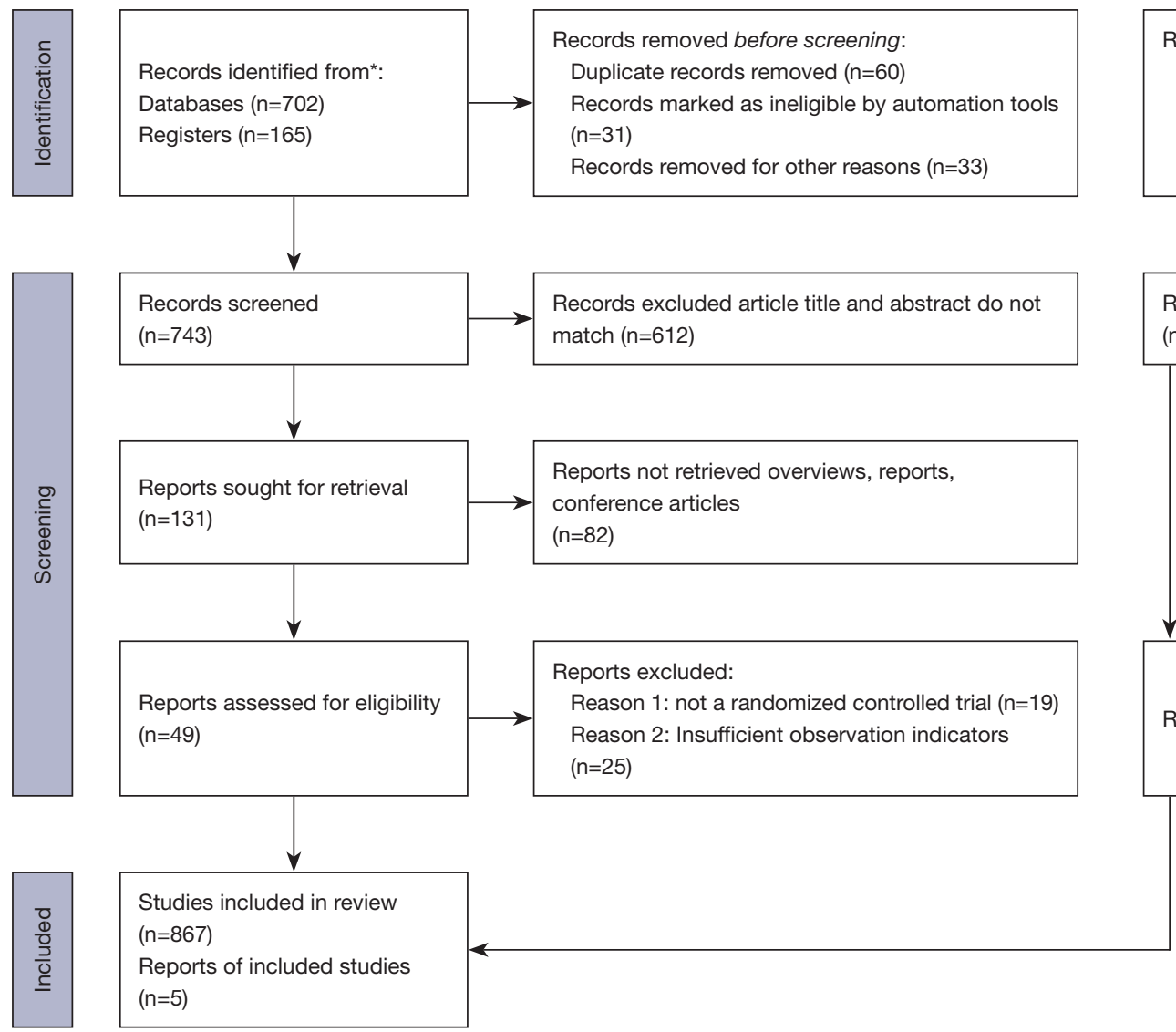

Records identified from:

Websites $(n=453)$

Organisations $(n=256)$

Citation searching $(n=158)$

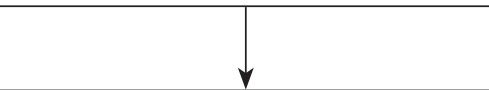

Reports sought for retrieval $(\mathrm{n}=867)$

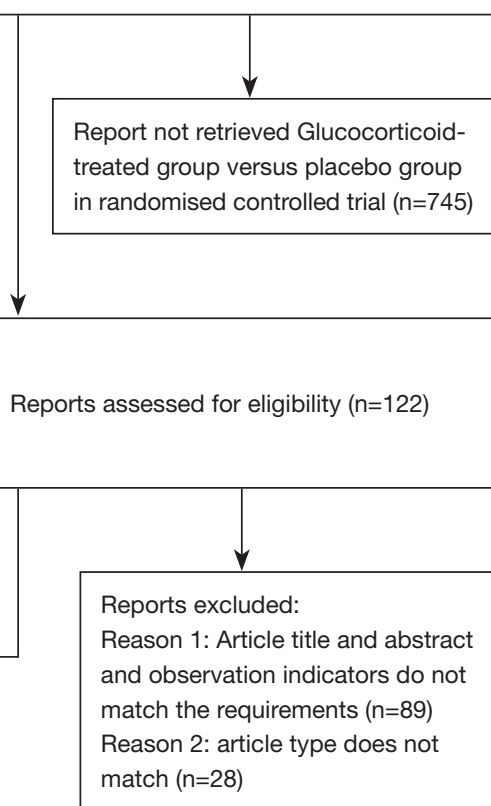

Figure 1 Literature search flowchart.

\section{Results}

\section{Search results and basic information of the included literature}

A total of 867 articles were obtained, and 743 articles were left after some articles were excluded. In addition, 612 articles were excluded after the article abstracts and titles were read, 82 articles were excluded because they were review articles and research reports, so 49 articles were left. Among them, 44 articles were excluded because the full texts were not randomized controlled experiments and articles with insufficient observation indicators. Finally, 5 articles were included in the study (Figure 1).

According to statistics, 5 (10-14) articles involving 217 patient cases met the inclusion criteria. In these 5 articles, all were small sample studies, with sample sizes ranging from 7 to 36 cases, and the age of the research subjects was 6 years old or above. All five articles described in detail the sample size, experimental grouping, treatment plan, treatment time, and efficacy indicators. The curative effect indicators included in the article were as follows: age at which the ability to walk was lost, age at which the ability to stand up from the ground was lost, muscle strength, muscle function, time to walk 9 meters, time to go up four floors, gowers time, quality of life, grip strength, time to walk 10 meters, weight lifting, leg function, forced vital capacity, creatine kinase level, time to walk 30 feet, and motor function and side effects. Table 1 showed the basic characteristics of the included articles.

\section{Results of the risk bias evaluation of the reference literature}

Figure 2 and Figure 3 showed the results of multiple risk bias 
Table 1 Basic characteristics of the included literature

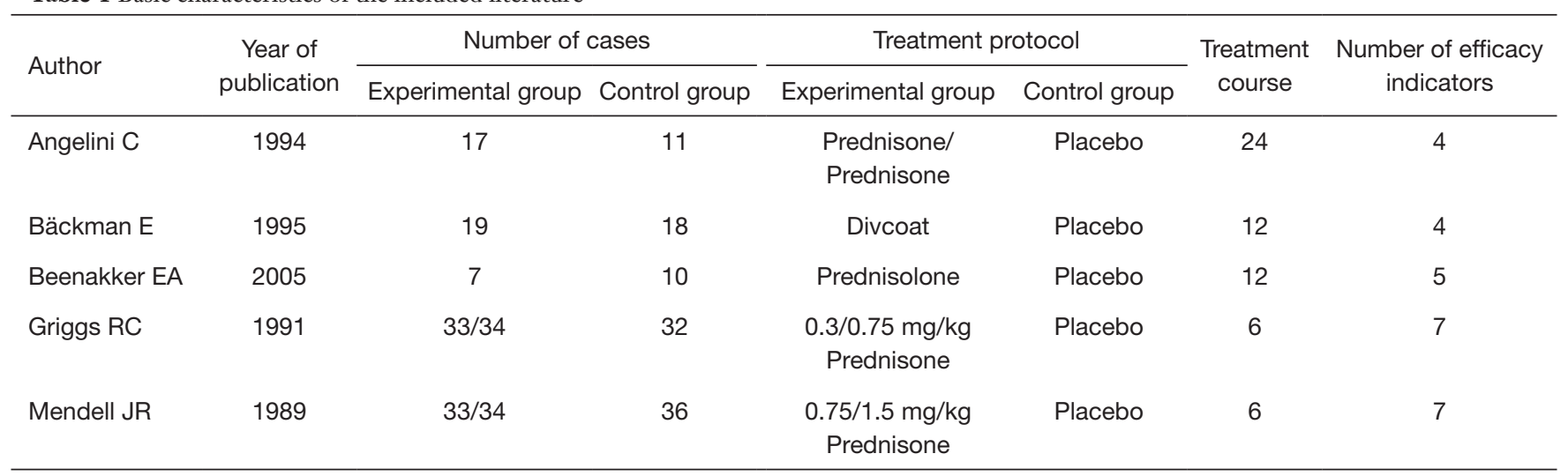

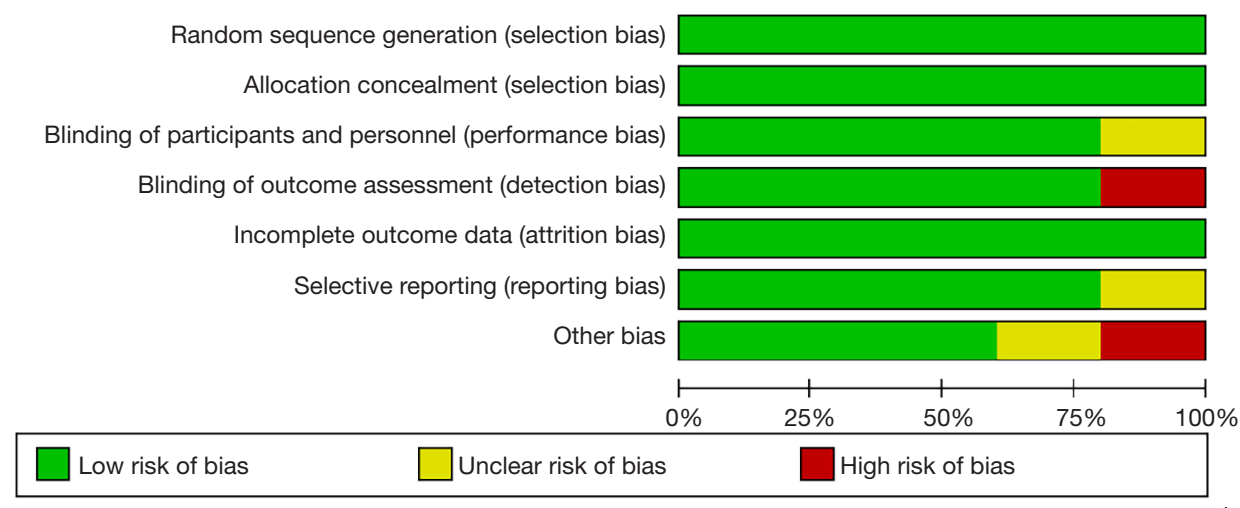

Figure 2 Results of risk bias evaluation of the included literature.

evaluations of the included articles drawn by RevMan 5.3 software. Among the 135 randomized controlled experiments in this study, 5 articles described the correct random allocation method, accounting for $100 \%$, and 34 articles described the concealed allocation plan in detail, accounting for $80 \%$; and there were 4 articles using blind method, accounting for $80 \%$, and no blind method was used in the remaining articles.

\section{Meta-analysis of the effect of glucocorticoids on mean muscle strength in PMD patients}

Figure 4 was a forest plot of the effect of glucocorticoids on the average muscle strength of PMD patients. Among the 5 articles included in the study, 3 articles described in detail the changes in the average muscle strength of patients in the glucocorticoid treatment group and the placebo control group. The relevant data were extracted from 3 articles to determine the average muscle strength of patients with PMD after treatment for heterogeneity analysis at the level. The results showed that chi-square test $\left(\mathrm{Chi}^{2}\right)=97.75$, degrees of freedom $(\mathrm{df})=2, \mathrm{I}^{2}=98 \%>50 \%$, and $\mathrm{P}<0.00001$, indicating that the average prognostic muscle strength of the two groups of PMD patients included in the articles showed an obvious heterogeneity. Statistical analysis using a random effects model found that the difference between the experimental group and the control group in the prognosis of PMD patients with glucocorticoid treatment was not statistically significant ( $\mathrm{MD}=1.77 ; 95 \% \mathrm{CI}$ : -0.95 to 4.48 ; $\mathrm{P}=0.20>0.05$ ).

\section{Meta-analysis of the effect of glucocorticoids on walking time of 9 meters in PMD patients}

Figure 5 was a forest plot of the effect of glucocorticoids on the walking time of 9 meters of PMD patients. Among the 


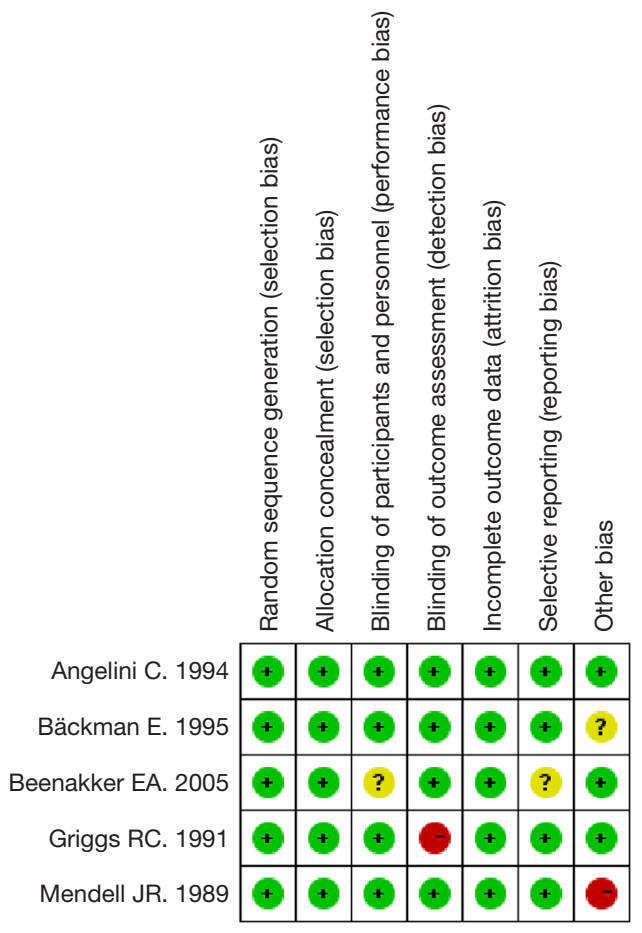

Figure 3 Results of multiple risk bias evaluations of the included studies. Note: + indicates low risk of bias; - indicates high risk of bias; ? indicates unclear.
5 articles included in the study, 4 articles described in detail the changes in the walking time of 9 meters of patients in the glucocorticoid treatment group and the placebo control group. The relevant data were extracted from 4 articles to determine the walking time of 9 meters of patients with PMD after treatment for heterogeneity analysis at the level. The results showed that $\mathrm{Chi}^{2}=379.39, \mathrm{df}=3, \mathrm{I}^{2}=99 \%>50 \%$, and $\mathrm{P}<0.00001$, indicating that the walking time of 9 meters of the two groups of PMD patients included in the articles showed an obvious heterogeneity. Statistical analysis using a random effects model found that the difference between the experimental group and the control group in the prognosis of PMD patients with glucocorticoid treatment was not statistically significant $(\mathrm{MD}=-12.27 ; 95 \% \mathrm{CI}:-35.94$ to $11.40 ; \mathrm{P}=0.31>0.01)$.

\section{Meta-analysis results of the effect of glucocorticoids on the time to go up four floors in PMD patients}

Figure 6 was a forest plot of the effect of glucocorticoids on the time to go up four floors of PMD patients. Among the 5 articles included in the study, 4 articles described in detail the changes in the time to go up four floors of patients in the glucocorticoid treatment group and the

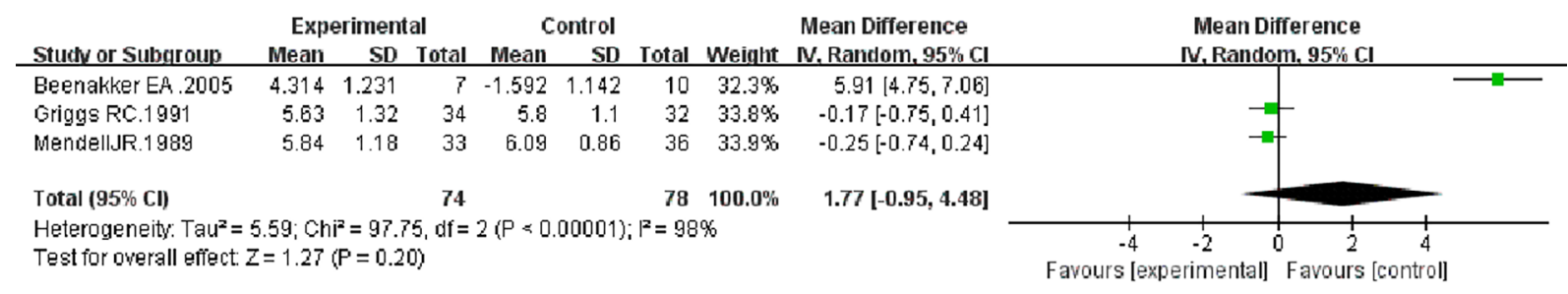

Figure 4 Forest plot of the effect of glucocorticoids on mean muscle strength in patients with PMD. PMD, progressive muscular dystrophy.

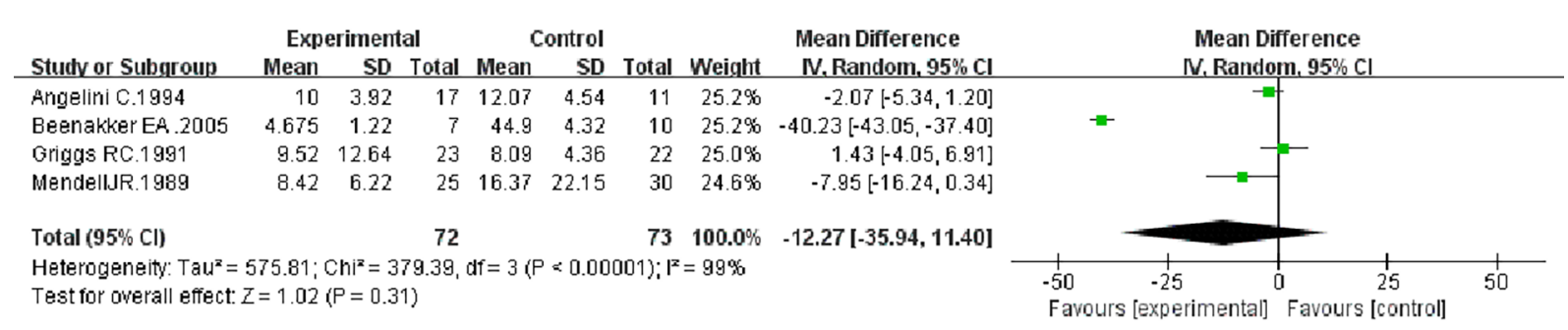

Figure 5 Forest plot of the effect of glucocorticoids on walking $9 \mathrm{~m}$ time in PMD patients. PMD, progressive muscular dystrophy. 


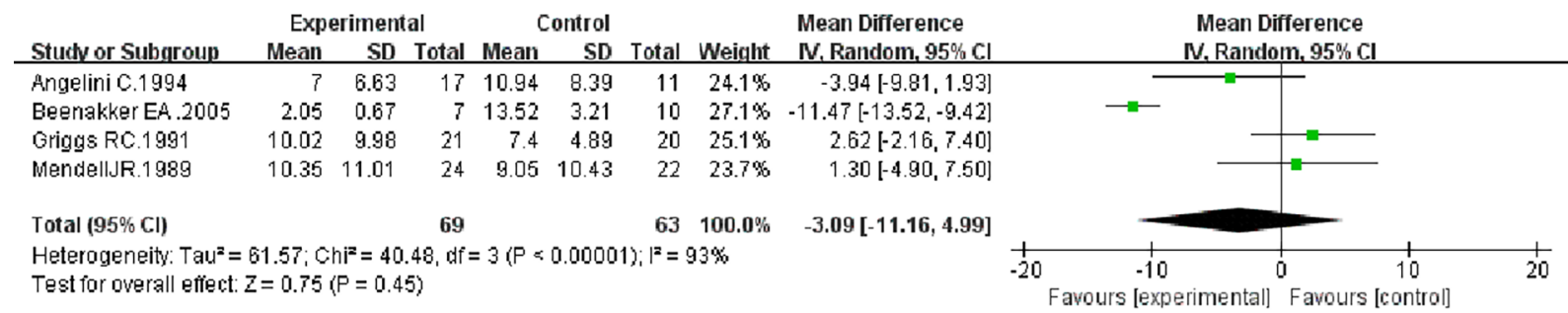

Figure 6 Forest plot of the effect of glucocorticoids on the time to climb four flights of stairs in PMD patients. PMD, progressive muscular dystrophy.

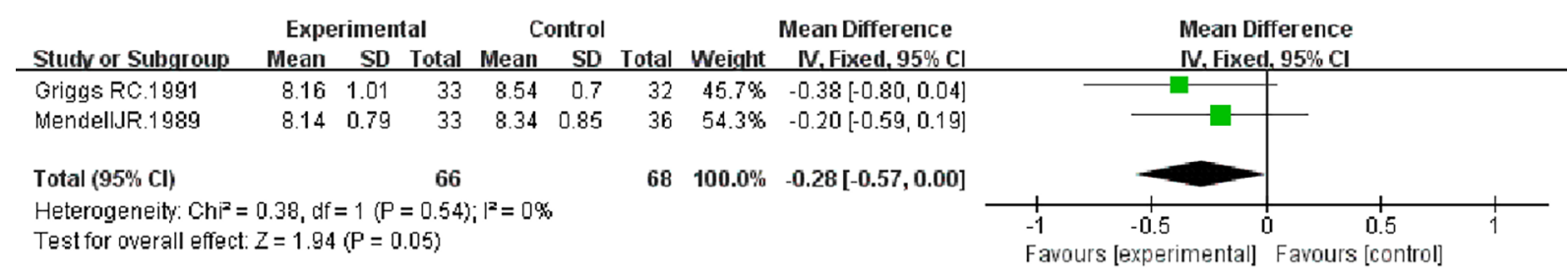

Figure 7 Forest plot of comparison on creatine kinase level.

placebo control group. The relevant data were extracted from 4 articles to determine the time to go up four floors of patients with PMD after treatment for heterogeneity analysis at the level. The results showed that $\mathrm{Chi}^{2}$ $=40.48, \mathrm{df}=3, \mathrm{I}^{2}=93 \%>50 \%$, and $\mathrm{P}<0.00001$, indicating that the time to go up four floors of the two groups of PMD patients included in the articles showed an obvious heterogeneity. Statistical analysis using a random effects model found that the difference between the experimental group and the control group in the time to go up four floors of PMD patients with glucocorticoid treatment was not statistically significant (MD $=-3.09 ; 95 \% \mathrm{CI}:-11.16$ to 4.99; $\mathrm{P}=0.45>0.05$ ).

\section{Meta-analysis of the effect of glucocorticoids on the level of creatine kinase in PMD patients}

Figure 7 was a forest plot of the effect of glucocorticoids on the level of creatine kinase of PMD patients. Among the 5 articles included in the study, 2 articles described in detail the changes in the level of creatine kinase of patients in the glucocorticoid treatment group and the placebo control group. The relevant data were extracted from 2 articles to determine the time to go up four floors of patients with PMD after treatment for heterogeneity analysis at the level. The results showed that $\mathrm{Chi}^{2}=0.38, \mathrm{df}=1, \mathrm{I}^{2}=0 \%<50 \%$, indicating that the level of creatine kinase of the two groups of PMD patients included in the articles showed no obvious heterogeneity. Statistical analysis using a fixed effects model found that the difference between the experimental group and the control group in the level of creatine kinase of PMD patients with glucocorticoid treatment was not statistically significant (MD $=-0.28 ; 95 \% \mathrm{CI}:-0.57$ to 0.00 ; $\mathrm{P}=0.05)$.

\section{Meta-analysis of the incidence of adverse reactions in patients with PMD treated with glucocorticoids}

The incidence of various adverse reactions in PMD patients after glucocorticoid treatment in the included articles was analyzed, and it was found that 4 of these 5 articles mentioned the incidence of adverse reactions in PMD patients after glucocorticoid treatment. The adverse reactions involved included patient behavior changes, short appearance, gastrointestinal problems, overgrowth of hair, acne, easy bruising, cataracts, diabetes, ankle edema, insomnia, anorexia, depression, hyperactivity, restlessness, irritability, excitement, increased appetite, weight gain/loss, and fatigue.

Among these adverse reactions, acne, excessive hair growth, irritability, and mild depression are symptoms that have been repeatedly mentioned. In response to this situation, detailed information describing the adverse reactions of PMD patients after glucocorticoid treatment 


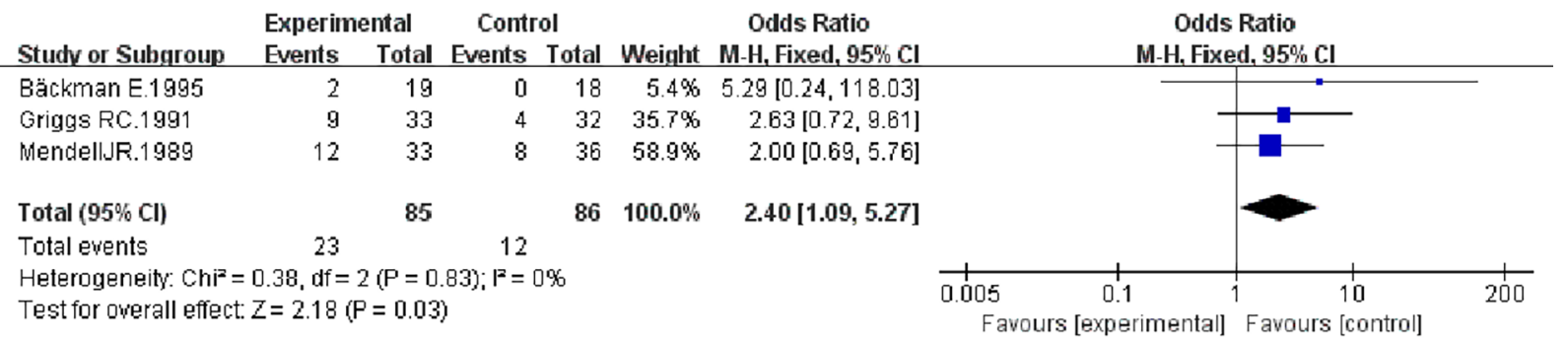

Figure 8 Forest diagram of the effect of glucocorticoid treatment on the incidence of acne in PMD patients. PMD, progressive muscular dystrophy.

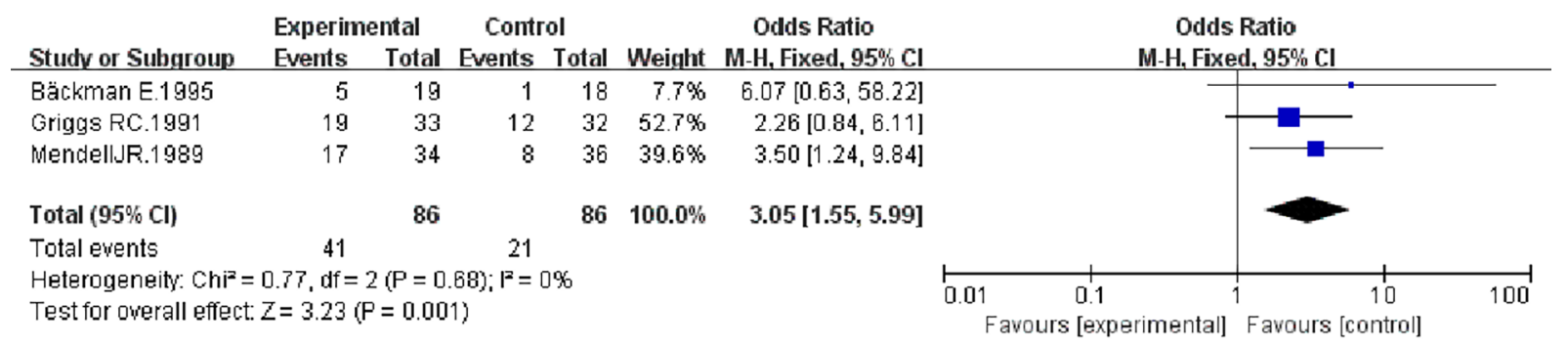

Figure 9 Forest diagram of the effect of glucocorticoid treatment on the incidence of PMD patients with excessive hair growth symptoms. PMD, progressive muscular dystrophy.

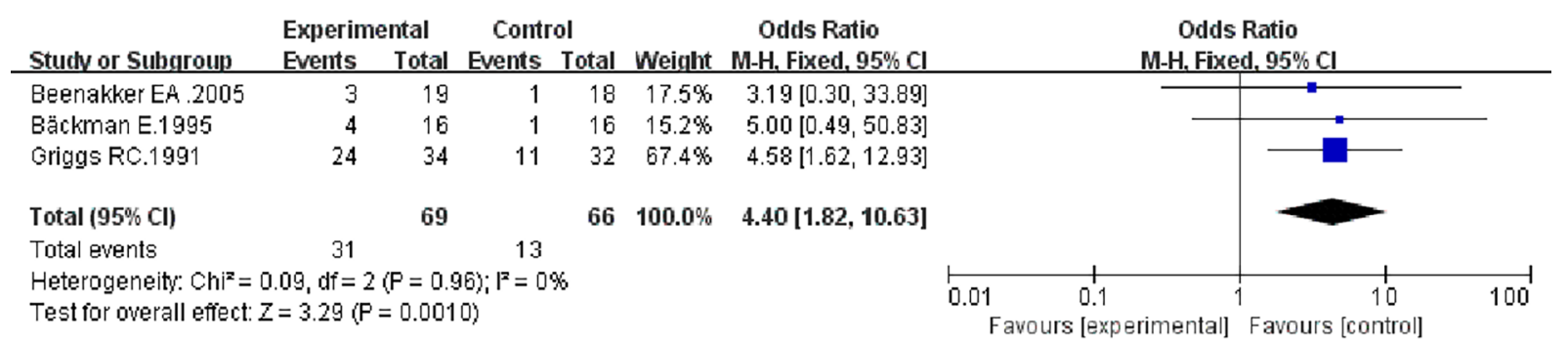

Figure 10 Forest plot of the effect of glucocorticoid therapy on the incidence of dysphoria in PMD patients. PMD, progressive muscular dystrophy.

of these 4 articles were extracted. It was found that among the 4 articles selected, 3 articles described in detail the incidence of acne in the prognosis of PMD patients. There were 3 articles describing in detail the incidence of excessive hair growth in the prognosis of PMD patients, 3 articles describing the incidence of irritability in the prognosis of PMD patients, and 3 articles describing in detail the incidence of depression in the prognosis of PMD patients. The relevant data were extracted to analyze the heterogeneity of the incidence of these 4 kinds of adverse reactions.
Figures 8-11 showed the comparison charts of the incidence of these four adverse reactions in PMD patients after glucocorticoid treatment. Among them, Figure 8 was a forest diagram of the effect of glucocorticoid treatment on the incidence of acne in PMD patients, Figure 9 was a forest diagram of the effect of glucocorticoid treatment on the incidence of PMD patients with excessive hair growth symptoms, Figure 10 was a forest plot of the effect of glucocorticoid therapy on the incidence of dysphoria in PMD patients, and Figure 11 was a forest plot of the effect of glucocorticoid therapy on the incidence of depression in 


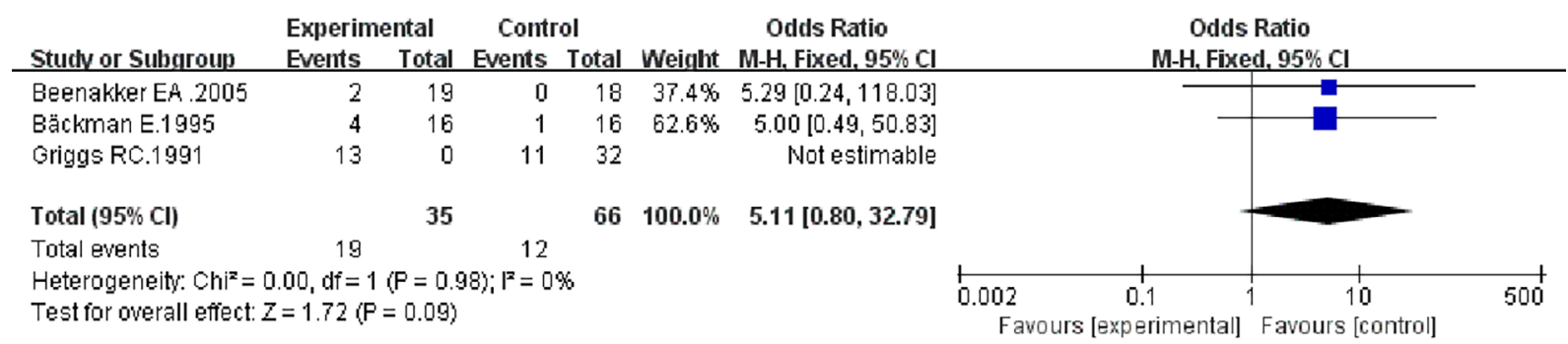

Figure 11 Forest plot of the effect of glucocorticoid therapy on the incidence of depression in PMD patients. PMD, progressive muscular dystrophy.

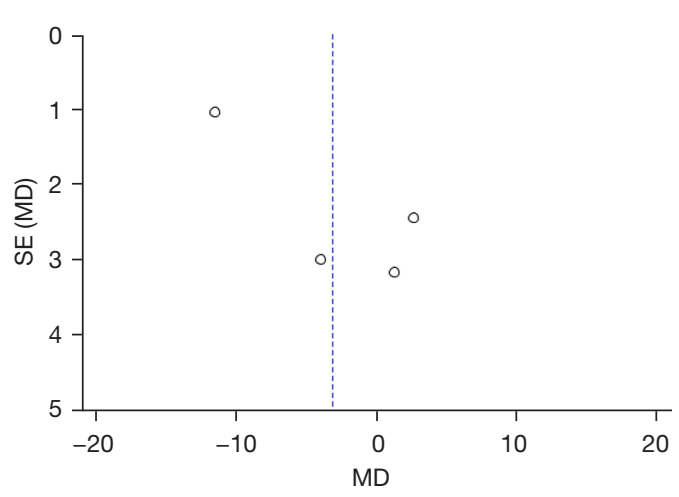

Figure 12 Climbing the funnel chart of four levels of time

PMD patients.

The heterogeneity of the incidence of acne between the glucocorticoid treatment group (experimental group) and the placebo treatment group (control group) (Figure 8) showed that $\mathrm{Chi}^{2}=0.38$, df $=2, \mathrm{I}^{2}=0 \%<50 \%, \mathrm{P}=0.83$, indicating that there was no obvious heterogeneity between the acne incidence between the experimental group and the control group included in the articles. Statistical analysis using a fixed-effects model found that compared with the control group, the probability of acne in PMD patients in the experimental group increased significantly, and the difference was statistically significant. It meant that glucocorticoids can significantly increase the prognostic risk of acne in PMD patients ( $\mathrm{OR}=2.40 ; 95 \%$ CI: 1.09 to 5.27; $\mathrm{P}=0.03<0.05)$.

The heterogeneity of the incidence of excessive hair growth symptoms between the experimental group and the control group (Figure 9) showed that $\mathrm{Chi}^{2}=0.77$, df $=2, I^{2}=0 \%<50 \%$, and $\mathrm{P}=0.68$, that there was no obvious heterogeneity between the experimental group and the control group in the prognosis of excessive hair growth symptoms. Statistical analysis using a fixed-effects model found that compared with the control group, the PMD patients in the experimental group had a significantly higher probability of developing symptoms of excessive hair growth, and the difference was statistically significant. It suggested that glucocorticoids can significantly increase the risk of excessive hair growth in the prognosis of PMD patients $(\mathrm{OR}=3.05 ; 95 \% \mathrm{CI}: 1.55$ to $5.99 ; \mathrm{P}=0.001<0.05)$.

The results of heterogeneity in the incidence of prognostic dysphoria symptoms between the experimental group and the control group (Figure 10) showed that $\mathrm{Chi}^{2}=0.09, \mathrm{df}=2, \mathrm{I}^{2}=0 \%<50 \%$, and $\mathrm{P}=0.96$. It showed that there was no obvious heterogeneity between the prognosis of patients in the experimental group and the control group. Statistical analysis using a fixed-effects model found that compared with the control group, the probability of experiencing emotional dysphoria in the experimental group of PMD patients increased significantly, and the difference was statistically significant. It suggested that glucocorticoids can significantly increase the risk of emotional dysphoria in the prognosis of $\mathrm{PMD}$ patients $(\mathrm{OR}=4.04,95 \% \mathrm{CI}: 1.82$ to $10.63 ; \mathrm{P}=0.001<0.05)$.

The heterogeneity of the incidence of depression between the experimental group and the control group (Figure 11) showed that $\mathrm{Chi}^{2}=0.00, \mathrm{df}=2, \mathrm{I}^{2}=0 \%<50 \%$, $\mathrm{P}=0.98$, indicating that there was no obvious heterogeneity between the experimental group and the control group in the incidence of depression. Statistical analysis using a fixed-effects model showed that there was no significant difference in the prognosis of depression between the experimental group and the control group for PMD patients $(\mathrm{OR}=5.11 ; 95 \% \mathrm{CI}: 0.80$ to $32.79 ; \mathrm{P}=0.09>0.05)$.

\section{Publication bias results}

Figures 12-14 showed the funnel charts of the publication bias of the included articles. Among them, Figures 12-14 


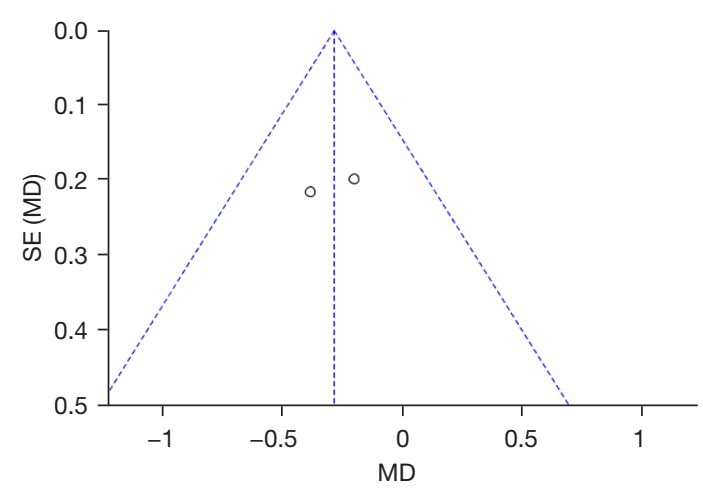

Figure 13 Funnel chart of creatine kinase levels.

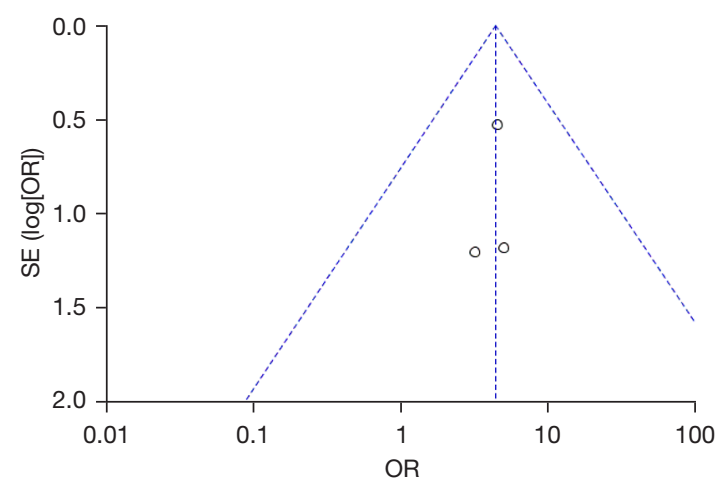

Figure 14 Funnel chart of the incidence of adverse reactions

were funnel diagrams drawn based on the information included in the articles on the time to climb 4 layers, the level of creatine kinase in the patient, and the incidence of patient adverse reactions, respectively. The figures illustrated that the circles in some of the included studies are basically concentrated on the midline and were roughly symmetrical to the midline, indicating that there was no bias in the publication of the included articles, and the conclusions drawn were relatively reliable.

\section{Discussion}

A total of 5 articles were included in this study for a systematic review, with the purpose of evaluating the efficacy and safety of glucocorticoids in patients with progressive muscular dystrophy. Among all the included articles, 5 articles described the correct random allocation method, 4 articles described the concealment of the allocation plan in detail, and 4 articles used the blind method. The reason may be the unclear random allocation method of the study and the subjective bias of doctors (15).

The results of this study showed that, compared with the control group, glucocorticoid treatment can improve the average muscle strength level of PMD patients, but there was a certain difference among the three included articles about the average muscle strength level of the prognosis of PMD patients treated with glucocorticoid Heterogeneity. The analysis results showed that there was no obvious difference in the prognostic average muscle strength between glucocorticoid treatment and placebo treatment of PMD patients [MD $=1.77,95 \% \mathrm{CI}:-0.95$ to 4.48; $\mathrm{P}=0.20>0.05]$. In addition, the heterogeneity analysis of 9-meter walking time in PMD patients showed that there was no significant difference in the prognosis of 9-meter walking time between glucocorticoid treatment and placebo-treated PMD patients ( $M D=-12.27$; 95\% CI: -35.94 to $11.40 ; \mathrm{P}=0.31>0.01)$. Similarly, the heterogeneity analysis of the time to climb the four stairs in PMD patients showed that there was no significant difference in the prognosis of the time to climb the four stairs in patients treated with corticosteroids and placebo (MD $=-3.09$; $95 \%$ CI: -11.16 to $4.99 ; \mathrm{P}=0.45>0.05$ ). The glucocorticoids and microbial agents mentioned in many reports have good curative effects in the treatment of PMD. It can extend the patient's independent walking time to a large extent, enhance the patient's muscle function and average muscle strength, and significantly alleviate the symptoms of low weight caused by malnutrition in patients with progressive muscular dystrophy (16-18). There are some discrepancies between the results of this study and the conclusions of the above studies. The reason for this phenomenon may be due to the fact that the number of relevant documents included in this study is too small; and there is a certain degree of bias between the results of different documents, which leads to a certain error in the difference analysis results of related indicators.

In addition, this study analyzed the heterogeneity of creatine kinase levels in the prognosis of PMD patients treated with glucocorticoid and placebo. The results showed that glucocorticoid treatment significantly reduced the levels of creatine kinase in PMD patients, the difference was significant statistically $(\mathrm{MD}=-0.28 ; 95 \%$ CI: -0.57 to $0.00 ; \mathrm{P}=0.05)$. Such results are consistent with the results of Escolar et al. (19). Studies have confirmed that glucocorticoid therapy can relieve the symptoms of abnormally elevated creatine kinase levels in patients with progressive muscular dystrophy, and reduce the risk of cardiomyopathy in patients with PMD (20). 
The results of heterogeneous analysis of the incidence of adverse reactions of glucocorticoid treatment in PMD patients showed that compared with the control group, the probability of acne in the experimental group of PMD patients increased significantly, and the difference was statistically significant. That is, glucocorticoids can significantly increase the prognostic risk of acne in PMD patients ( $\mathrm{OR}=2.40 ; 95 \%$ CI: 1.09 to $5.27 ; \mathrm{P}=0.03<0.05$ ). Compared with the control group, the PMD patients in the experimental group showed a significantly higher probability of having symptoms of excessive hair growth $(\mathrm{OR}=3.05$; $95 \%$ CI: 1.55 to $5.99 ; \mathrm{P}=0.001<0.05)$. The heterogeneity of the incidence of emotional dysphoria symptoms in the experimental group and the control group showed that glucocorticoids can significantly increase the risk of emotional dysphoria in the prognosis of PMD patients ( $\mathrm{OR}=4.04 ; 95 \% \mathrm{CI}: 1.82$ to $10.63 ; \mathrm{P}=0.001<0.05)$. The results of the heterogeneity of the incidence of prognostic depression between the experimental group and the control group showed that there was no significant difference in the prognosis of depression between the two groups of patients $(\mathrm{OR}=5.11 ; 95 \% \mathrm{CI}$ : 0.80 to $32.79 ; \mathrm{P}=0.09>0.05)$. The above results confirm that glucocorticoid therapy will significantly increase the probability of adverse prognosis for patients compared with placebo therapy. Among them, the probability of adverse reactions such as acne, rapid hair growth, and emotional irritability is significantly increased. In other related studies, it is also mentioned that during the clinical use of glucocorticoids, some complications will occur, such as obesity, hirsutism, acne, short stature, delayed puberty, abnormal behavior, immunosuppression, hypertension, impaired glucose tolerance, gastrointestinal symptoms, cataracts, osteoporosis, vertebral compression fractures, and long bone fractures $(21,22)$.

The funnel chart of the meta-analysis of the efficacy and safety of glucocorticoids in PMD patients showed that the published articles selected in this study was not biased. Therefore, the conclusions obtained were more credible, and risk bias was not the main factor affecting the conclusions.

\section{Conclusions}

This study included 5 papers related to the clinical glucocorticoid treatment in patients with PMD. We employed meta-analysis to confirm that glucocorticoids could markedly improve the mean muscle strength and muscle function of patients, which can significantly prolong the independent walking time and quality of life of patients.
Yet, there are certain safety concerns, such as adverse effects including behavioral changes, hirsutism, Cushing's face, weight changes, hypertension, and osteoporosis, that which occur in a certain percentage of patients after treatment. The limitations of this study were as follows: (I) the lack of information on clinical data for infants and children under 6 years of age in the literature; (II) the small sample sizes of the included studies; (III) the large error of their results of the included studies; (IV) there are differences in the types and doses of glucocorticoids used in each study, resulting in information bias in the data collection process; and (V) by changing the inclusion criteria and excluding lowquality studies, we were not able to significantly reduce the heterogeneity. There was a certain amount of heterogeneity, which may have had some influence on the results. Further studies are needed in future on patients with low age PMD and PMD patients who cannot walk independently. In conclusion, this study provides some scientific reference for the efficacy assessment and safety evaluation of glucocorticoid therapy in PMD patients.

\section{Acknowledgments}

Funding: This study was supported by the Xinjiang Uygur Autonomous Region Natural Science Fund (2019D01C310).

\section{Footnote}

Reporting Checklist: The authors have completed the PRISMA reporting checklist. Available at https://dx.doi. org/10.21037/tp-21-461

Conflicts of Interest: All authors have completed the ICMJE uniform disclosure form (available at https://dx.doi. org/10.21037/tp-21-461). The authors have no conflicts of interest to declare.

Ethical Statement: The authors are accountable for all aspects of the work in ensuring that questions related to the accuracy or integrity of any part of the work are appropriately investigated and resolved.

Open Access Statement: This is an Open Access article distributed in accordance with the Creative Commons Attribution-NonCommercial-NoDerivs 4.0 International License (CC BY-NC-ND 4.0), which permits the noncommercial replication and distribution of the article with 
the strict proviso that no changes or edits are made and the original work is properly cited (including links to both the formal publication through the relevant DOI and the license). See: https://creativecommons.org/licenses/by-nc-nd/4.0/.

\section{References}

1. Li M, Cai Y, Zhong M, et al. A novel treatment regimen for Duchenne muscular dystrophy. Neuroreport 2013;24:924-7.

2. Hoffman EP, Schwartz BD, Mengle-Gaw LJ, et al. Vamorolone trial in Duchenne muscular dystrophy shows dose-related improvement of muscle function. Neurology 2019;93:e1312-23.

3. Guglieri M, Bushby K, McDermott MP, et al. Developing standardized corticosteroid treatment for Duchenne muscular dystrophy. Contemp Clin Trials 2017;58:34-9.

4. Griggs RC, Miller JP, Greenberg CR, et al. Efficacy and safety of deflazacort vs prednisone and placebo for Duchenne muscular dystrophy. Neurology 2016;87:2123-31.

5. Buyse GM, Voit T, Schara U, et al. Treatment effect of idebenone on inspiratory function in patients with Duchenne muscular dystrophy. Pediatr Pulmonol 2017;52:508-15.

6. McDonald CM, Henricson EK, Abresch RT, et al. The 6-minute walk test and other endpoints in Duchenne muscular dystrophy: longitudinal natural history observations over 48 weeks from a multicenter study. Muscle Nerve 2013;48:343-56.

7. Victor RG, Sweeney HL, Finkel R, et al. A phase 3 randomized placebo-controlled trial of tadalafil for Duchenne muscular dystrophy. Neurology 2017;89:1811-20.

8. Rutter MM, Wong BL, Collins JJ, et al. Recombinant human insulin-like growth factor-1 therapy for 6 months improves growth but not motor function in boys with Duchenne muscular dystrophy. Muscle Nerve 2020;61:623-31.

9. Jensen L, Petersson SJ, Illum NO, et al. Muscular response to the first three months of deflazacort treatment in boys with Duchenne muscular dystrophy. J Musculoskelet Neuronal Interact 2017;17:8-18.

10. Angelini C, Pegoraro E, Turella E, et al. Deflazacort in Duchenne dystrophy: study of long-term effect. Muscle Nerve 1994;17:386-91. Erratum in: Muscle Nerve 1994;17:833.

11. Bngelini C, Pegoraro E, Turella E, et al. Deflazacort in Duchenne dystrophy: study of long-tstrophy. Neuromuscul Disord 1995;5:233-41.

12. Beenakker EA, Fock JM, Van Tol MJ, et al. Intermittent prednisone therapy in Duchenne muscular dystrophy: a randomized controlled trial. Arch Neurol 2005;62:128-32.

13. Griggs RC, Moxley RT 3rd, Mendell JR, et al. Prednisone in Duchenne dystrophy. A randomized, controlled trial defining the time course and dose response. Clinical Investigation of Duchenne Dystrophy Group. Arch Neurol 1991;48:383-8.

14. Mendell JR, Moxley RT, Griggs RC, et al. Randomized, double-blind six-month trial of prednisone in Duchenne's muscular dystrophy. N Engl J Med 1989;320:1592-7.

15. Hendell JR, Moxley RT, Griggs RC, et al. Randomized, double-blind six-month trial of prednisone in Duchenne's muscular dystrophy. N Engl J Med 1989;320cocorticoids. Amino Acids 2015;47:1853-63.

16. Hussein MR, Abu-Dief EE, Kamel NF, et al. Steroid therapy is associated with decreased numbers of dendritic cells and fibroblasts, and increased numbers of satellite cells, in the dystrophic skeletal muscle. J Clin Pathol 2010;63:805-13.

17. Kao KT, Joseph S, Capaldi N, et al. Skeletal disproportion in glucocorticoid-treated boys with Duchenne muscular dystrophy. Eur J Pediatr 2019;178:633-40.

18. Mayer OH, Leinonen M, Rummey C, et al. Efficacy of Idebenone to Preserve Respiratory Function above Clinically Meaningful Thresholds for Forced Vital Capacity (FVC) in Patients with Duchenne Muscular Dystrophy. J Neuromuscul Dis 2017;4:189-98.

19. Escolar DM, Hache LP, Clemens PR, et al. Randomized, blinded trial of weekend vs daily prednisone in Duchenne muscular dystrophy. Neurology 2011;77:444-52.

20. McDonald CM, Meier T, Voit T, et al. Idebenone reduces respiratory complications in patients with Duchenne muscular dystrophy. Neuromuscul Disord 2016;26:473-80.

21. Hu J, Ye Y, Kong M, et al. Daily prednisone treatment in Duchenne muscular dystrophy in southwest China. Muscle Nerve 2015;52:1001-7.

22. Mavroudis PD, van den Anker J, Conklin LS, et al. Population Pharmacokinetics of Vamorolone (VBP15) in Healthy Men and Boys With Duchenne Muscular Dystrophy. J Clin Pharmacol 2019;59:979-88.

(English Language Editor: A. Kassem)

Cite this article as: Ru L, Wang Y, Yan M. Efficacy and safety of glucocorticoids in the treatment of progressive muscular dystrophy in children: a systematic review and meta-analysis. Transl Pediatr 2021;10(11):3046-3057. doi: 10.21037/tp-21-461 